Classification

Physics Abstracts

$77.20-77.30-77.40-77.55-77.90$

\title{
Les polymères thermostables pour l'électronique
}

\author{
A. Bui, J. Farenc et Y. Segui \\ Laboratoire de Génie Electrique, 118, Route de Narbonne, 31062 Toulouse Cedex, France
}

(Reçu le 20 février 1986, accepté le 7 juillet 1986)

\begin{abstract}
Résumé. - Le présent article passe en revue les applications des polymères dans les industries électronique et électrotechnique puis donne pour chacune de ces applications les besoins en thermostables. Nous étudierons ensuite un cas particulier, celui de la protection rapprochée des composants au silicium et de l'isolation multicouche et nous présenterons les caractéristiques physiques et électriques que demande ce type d'applications. Enfin, nous donnerons les récents résultats de mesures électriques et thermiques obtenus sur deux variétés de polymères thermostables, le polyimide et le polyphénylquinoxaline, utilisables pour les applications particulières citées précédemment.
\end{abstract}

Abstract. - This paper describes the applications of thermostable polymers for the electronic and electrotechnic industries and point out the present need for these applications. A particular attention is paid to the cases of insulation for multilevel interconnection and primary passivation of silicon devices. Finally some experimental results on electrical and thermal behaviour of polyimide and polyphenylquinoxaline are reported.

\section{Introduction.}

Le développement d'un bon nombre de matières plastiques modernes a trouvé son origine dans les besoins exprimés par les Industries Electriques et Electroniques, principalement grâce aux nombreux champs d'application de ces matériaux que l'on peut trouver dans la fabrication des composants. Les principales raisons de cette popularité sont que la matière plastique est bon marché, facile à mettre en œuvre, avec un contrôle aisé des propriétés physiques, thermiques et électriques.

En ce qui concerne les polymères thermostables, leur développement ne fait que croître à cause de deux facteurs :

- la tendance des composants à évoluer vers une plus grande miniaturisation pour une même puissance dissipée ;

- l'utilisation d'un processus technologique impliquant une ou plusieurs étapes durant lesquelles la température du composant peut atteindre des températures importantes, telles que la soudure, le scellement ou la métallisation par évaporation sous vide.

Dans ce texte, nous allons aborder trois points principaux :

- passer en revue les applications des polymères dans les industries électronique et électrotechnique, puis donner pour chacune de ces applications les besoins en thermostables,
- en extraire un cas particulier, celui de la protection rapprochée des composants au silicium et de l'isolation multicouche et présenter les caractéristiques physiques et électriques que demande ce type d'applications,

- donner les récents résultats obtenus sur deux variétés de polymères thermostables, le polyimide et le polyphénylquinoxaline, utilisables pour les applications particulières citées précédemment.

\section{Principales utilisations des polymères thermosta- bles en électronique et électrotechnique.}

En ce qui concerne l'Electrotechnique, c'est-à-dire l'électricité des courants forts et de la haute tension, les polymères thermostables peuvent entrer dans les domaines suivants :

- les câbles de transport d'énergie électrique à haute tension, les condensateurs de puissance,

- les machines tournantes, c'est-à-dire les alternateurs et les moteurs. Pour ce type de matériel, il est intéressant d'avoir des polymères pouvant supporter des températures égales ou supérieures à $300{ }^{\circ} \mathrm{C}$ dans le but d'augmenter les puissances volumiques et massiques.

En ce qui concerne l'Electronique, c'est-à-dire l'électricité des courants faibles, on peut distinguer trois domaines de pénétration des polymères :

- Le cas où le polymère est utilisé au cours de la fabrication du composant électronique et ensuite 
totalement éliminé. Il s'agit essentiellement des liants organiques utilisés dans les encres sérigraphiques et les laques photosensibles destinées à la gravure.

- Le cas où le produit organique est utilisé pour former une barrière de protection de la surface active du composant contre les attaques chimiques ou les atteintes d'origine mécanique. Cette protection peut être très rapprochée c'est le cas des couches de passivation auxquelles on demande en plus, dans certaines applications, des qualités d'isolation électrique pour séparer les couches de métallisation d'interconnexion. Le cas de la protection éloignée est celui des boîtiers et de l'encapsulation plastique dont l'emploi est maintenant généralisé dans le domaine des applications industrielles et grand public.

- Le cas où la matière plastique participe à la structure du composant ou du système électronique par ses propriétés électriques ou mécaniques. Dans cette catégorie, la consommation du produit est, de loin, la plus importante. On peut citer par exemple :

2.1 LES CONNECTEURS. - Les polymères utilisés dans cette fabrication doivent satisfaire les exigences suivantes :

- stabilité dimensionnelle, à cause de la réduction de l'intervalle qui sépare deux contacts,

- résistance à la chaleur et aux attaques chimiques ; le connecteur est soumis à la température de la soudure et aux solvants organiques utilisés pendant cette opération,

- bonnes propriétés électriques ; il est à noter que les applications haute fréquence, qui deviennent de plus en plus importantes exigent du matériau une faible valeur du facteur de pertes et de la permittivité relative. Les matériaux que l'on utilise couramment sont le polycarbonate, le polybutylène téréphthalate et le polysulfone. Pour les connecteurs devant supporter $200{ }^{\circ} \mathrm{C}$ en service continu, le poly(sulfure de phénylène) peut être utilisé mais est d'un coût élevé.

2.2 LES CIRCUITS IMPRIMÉS. - On distingue trois types de circuits imprimés :

- le circuit simple face : les conducteurs sont sur une seule face du substrat isolant,

- le circuit double face : les conducteurs sont sur les deux faces du substrat isolant,

- le circuit multicouche : plusieurs couches de conducteurs sont séparées par des couches isolantes.

La plupart des circuits imprimés sont réalisés à l'aide de matériaux thermodurcissables. Il s'agit des époxys, des polyimides et des résines phénoliques. Pour les circuits microondes, on utilise des substrats à base de polytétrafluoroéthylène à cause de la très faible valeur des pertes diélectriques de ce produit.

En ce qui concerne les thermoplastiques, ce sont les thermostables du type polysulfone, poly(sulfure de phénylène) et polyéther-imide qui sont utilisés.

2.3 LeS CONDENSATEURS. - Pour des applications à température normale, les condensateurs sont réalisés à l'aide de films de polystyrène, de polycarbo- nate, de polypropylène ou de polyéthylène téréphthalate. Pour les applications à haute température on utilise des matériaux tels que le polytétrafluoroéthylène ou le polyimide.

2.4 LES MATÉRIAUX POUR LA FIXATION DE LA " PUCE » SUR LE SUBSTRAT. - Pour fixer la pastille de silicium sur l'embase céramique, avant le scellement de cette dernière au capot, on utilise un eutectique $\mathrm{Si}-\mathrm{Au}$. Pour réduire le coût de cette technique, des essais ont été réalisés avec des composites polymères thermostables/argent. Les résultats obtenus sont encourageants.

2.5 LA PROTECTION RAPPROCHÉE ET L'ISOLATION MULTICOUCHE. - Les couches de passivation des composants au silicium peuvent être classées en deux catégories :

- les couches de passivation primaire qui sont directement en contact avec le cristal de silicium sur lequel sont réalisés les composants,

- les couches de passivation secondaire qui sont séparées du silicium par une couche de diélectrique et/ou par une couche de métallisation. La double fonction de cette couche de diélectrique est d'arrêter la diffusion d'ions alcalins et de servir de support diélectrique pour la réalisation des interconnexions.

\section{Caractéristiques essentielles des produits de passi- vation.}

La séquence classique de dépôt des couches de passivation sur le cristal de silicium et la nature de ces couches varient suivant le type de composant. Mais d'une manière générale, le processus est le suivant :

$$
\text { Passivation primaire }\left(\mathrm{SiO}_{2}\right. \text { thermique) }
$$

Passivation secondaire (verre phosphosilicate, $\mathrm{Si}_{3} \mathrm{~N}_{4}$, $\mathrm{Al}_{2} \mathrm{O}_{3}$,

$$
\begin{gathered}
\text { Matériaux organiques) } \\
\text { Métallisation ( } \mathrm{Al}, \mathrm{Au}, \text { Réfractaires) } \\
\text { Passivation secondaire } \\
\text { (verre, } \mathrm{Si}_{3} \mathrm{~N}_{4}, \mathrm{SiO}_{2}, \text { Matériaux organiques) }
\end{gathered}
$$

Jusqu'à maintenant les matériaux organiques ne sont utilisés que pour certains types de composants, notamment les composants de puissance. Mais on pense que ces matériaux présentent un grand intérêt pour les circuits intégrés à large échelle qui exigent plusieurs couches isolantes pour les interconnexions.

Les deux avantages principaux des matériaux organiques par rapport aux inorganiques sont :

* la planarisation, c'est-à-dire la faculté d'obtenir des couches planes à partir d'un substrat non plan,

* la faible émission de particules alpha qu'il est important d'obtenir dans la fabrication de mémoires dynamiques. 
Les caractéristiques exigées d'un matériau de passivation pour composants à semi-conducteur peuvent être regroupées en deux catégories :

3.1 LES CARACTÉRISTIQUES PHYSIQUES OU PHYSICO-CHIMIQUES. - Stabilité thermique : durant sa fabrication et son montage dans le boîtier, le composant est soumis à des cycles de température qui peuvent atteindre des valeurs importantes : métallisation, soudures, collage sur le substrat, scellement du capot. Dans cette dernière opération on peut atteindre $450{ }^{\circ} \mathrm{C}$ pendant quelques minutes. Le matériau ne doit pas se dégrader mais de plus il ne doit pas rejeter des produits volatiles qui, enfermés dans le boîtier, mettent en cause la vie du composant.

- La stabilité dimensionnelle: les couches de passivation et d'isolant pour les interconnexions sont en contact intime avec d'autres matériaux généralement inorganiques ayant un coefficient de dilatation thermique différent. Les matériaux plastiques ont un coefficient de dilatation thermique plus grand que celui du métal et du silicium qu'il est important de réduire en travaillant au-dessous de $T_{\mathrm{g}}$ ou en augmentant le poids moléculaire par réticulation ou encore en jouant sur les groupes terminaux des chaînes moléculaires.

- La pureté de la résine et la propriété à l'humidité : l'absorption de l'humidité par le matériau et la présence, en son sein, d'impuretés ioniques conduisent à la corrosion électrochimique des conducteurs en aluminium et à la défaillance à terme du composant.

De ce point de vue l'adhésion du polymère sur les matériaux avec lesquels il est en contact est une propriété importante parce qu'elle empêche le transport de l'humidité par capillarité le long des interfaces jusqu'au cœur des éléments actifs.

- La planarisation: le dépôt de la couche de polymère sur le cristal se fait à l'aide d'un procédé habituellement utilisé pour déposer des couches de photorésistance. Il consiste à utiliser une solution à faible viscosité que l'on étale et évapore sur le cristal en le faisant tourner à grande vitesse. Cette couche est déposée sur des conducteurs métalliques présentant une marche. D'autre part, étant destinée à recevoir d'autres conducteurs métallisés, elle doit présenter une surface supérieure parfaitement plane. Ceci se réalise parfaitement à l'aide des polymères.

- L'émission de particules alpha: dans le cas des circuits à mémoire, les particules alpha émises par les traces d'éléments radioactifs contenus dans les matériaux de passivation, conduisent à des erreurs aléatoires. En effet, des paires électrons/trous produites peuvent modifier l'état logique de l'élément de mémoire. De ce point de vue les polymères présentent un avantage sur les matériaux inorganiques.

- La possibilité de prise de contact : le matériau de passivation ou d'isolation multicouche doit être facilement attaquable soit par une solution chimique, soit par un procédé plasma pour graver des trous à travers lesquels les contacts électriques sont établis entre les différentes métallisations.

3.2 LES PROPRIÉTÉS ÉLECTRIQUES. - Les propriétés diélectriques : il s'agit essentiellement de la permittivité relative du matériau et du facteur de perte diélectrique. Pour les polymères, la constante diélectrique est relativement plus faible que celle des matériaux inorganiques. Ce facteur constitue un avantage lorsqu'on a affaire à des circuits rapides.

- La conduction et la rupture électrique: la conduction, c'est-à-dire les caractéristiques couranttension et la rupture, c'est-à-dire la tension à partir de laquelle la couche de passivation perd ses propriétés d'isolation, sont deux caractéristiques essentielles pour la passivation et l'isolation multicouche.

- La caractéristique capacité-tension: lorsqu'on réalise des structures métal-isolant-semi-conducteur (MIS) et que l'on mesure leur capacité en fonction de la tension continue appliquée, on obtient une courbe $C(V)$ à partir de laquelle on peut obtenir deux grandeurs intéressant la stabilité du composant :

** la densité de charges à l'interface semi-conducteur-isolant- $Q_{\mathrm{T}}$,

** la densité des états de surface $N_{\mathrm{ss}}$.

La grandeur de ces deux paramètres et surtout leur évolution dans le temps conduisent à une dérive des caractéristiques électriques du composant à cause de la couche d'inversion qu'ils créent à la surface du cristal.

4. Principaux résultats obtenus sur le polyimide et sur le polyphénylquinoxaline.

4.1 Formules CHIMIQUes. - Pour les polyamidesimides, la formule chimique est de la forme :

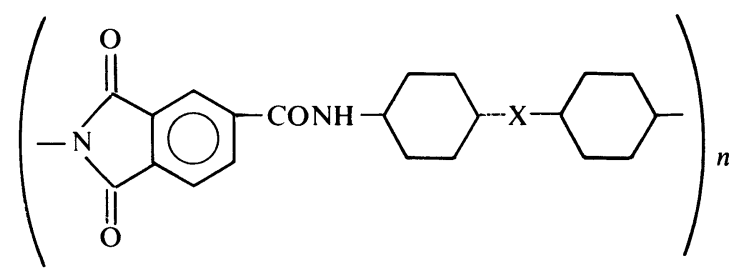

où $x$ peut être soit un atome d'oxygène, soit un groupement $\mathrm{CH}_{2}$. Pour les polyphénylquinoxalines la formule chimique est :

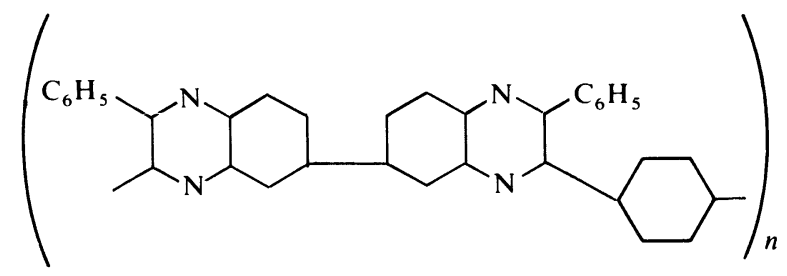

4.2 PRINCIPAUX RÉSUltats. - On a regroupé dans le tableau I les principales caractéristiques électriques et physiques des deux polymères thermostables et celles obtenues à partir de la silice thermique. 
Tableau I. - Comparaison des propriétés électriques et thermiques de la silice de polymères thermostables.

[Comparison of electrical and thermal properties of $\mathrm{SiO}_{2}$ and thermostable polymers.]

\begin{tabular}{|c|c|c|c|}
\hline & $\mathrm{SiO}_{2}$ & Polyamide-imide & Polyphénylquinoxaline \\
\hline$\varepsilon$ & 4,2 & 3,5 & 3,6 \\
\hline $\operatorname{tg} \delta$ & $\mathrm{qq} \times 10^{-4}$ & $\mathrm{qq} \times 10^{-3}$ & $\mathrm{qq} \times 10^{-3}$ \\
\hline $\begin{array}{c}\text { Rigidité } \\
\text { diélectrique }\end{array}$ & $10^{6}-10^{7} \mathrm{~V} / \mathrm{cm}$ & $10^{6} \mathrm{~V} / \mathrm{cm}$ & $2 \times 10^{6} \mathrm{~V} / \mathrm{cm}$ \\
\hline$\rho$ & $10^{16} \Omega . \mathrm{cm}$ & $\begin{array}{l}10^{17} \Omega . \mathrm{cm} \text { à } 20^{\circ} \mathrm{C} \\
10^{14} \Omega . \mathrm{cm} \text { à } 150^{\circ} \mathrm{C}\end{array}$ & $3 \times 10^{17} \Omega . \mathrm{cm}$ à $20^{\circ} \mathrm{C}$ \\
\hline$Q_{\mathrm{T}}$ & $2 \times 10^{10} \mathrm{~cm}^{-2}$ & $10^{11} \mathrm{~cm}^{-2}$ & - \\
\hline$N_{\text {ss }}$ & $10^{10}$ à $10^{12} \mathrm{~cm}^{-2} \cdot \mathrm{eV}^{-1}$ & $5 \times 10^{11}$ à $5 \times 10^{12} \mathrm{~cm}^{-2} \cdot \mathrm{eV}^{-1}$ & 一 \\
\hline $\begin{array}{l}\text { Dégradation } \\
\text { thermique }\end{array}$ & & 400 à $450^{\circ} \mathrm{C}$ & 450 à $500{ }^{\circ} \mathrm{C}$ \\
\hline $\begin{array}{l}\text { Dilatation } \\
\text { thermique }\end{array}$ & 3 à $5 \times 10^{-6} / \mathrm{o}$ & 2 à $4 \times 10^{-5} / \mathrm{o}$ & 5 à $6 \times 10^{-5} / \mathrm{o}$ \\
\hline$T_{\mathrm{g}}$ & & $340^{\circ} \mathrm{C}$ & $340^{\circ} \mathrm{C}$ \\
\hline
\end{tabular}

En ce qui concerne les grandeurs électriques, on peut faire les observations suivantes :

- La permittivité relative est de l'ordre de 3,5 pour le polyamide et le polyphénylquinoxaline. Elle est légèrement inférieure à celle de la silice, ce qui présente un avantage pour les polymères bien que le facteur de pertes diélectriques soit nettement plus favorable pour la silice. La rigidité diélectrique de la silice est d'un ordre de grandeur supérieure à celle des polymères. Mais ce n'est pas un facteur déterminant car le niveau de tension mis en jeu dans les circuits intégrés est relativement faible.

- On n'a pas encore déterminé les valeurs de $Q_{\mathrm{T}}$ et $N_{\mathrm{ss}}$ du polyphénylquinoxaline. Mais la comparaison des résultats obtenus avec le polyamide a permis de voir qu'ils ne sont pas très éloignés de ceux de la silice et que la stabilité de $Q_{\mathrm{T}}$ du polyamide est très bonne en fonction du temps de polarisation.

- La dégradation thermique du polyphénylquinoxaline se produit à une température de $500^{\circ} \mathrm{C}$, c'est-à-dire environ $50^{\circ} \mathrm{C}$ au-dessus de celle du polyamide.

\section{Conclusion.}

Le tableau précédent montre que si la plupart des propriétés électriques, thermiques et mécaniques des polymères thermostables ont pu être déterminées, il serait nécessaire de poursuivre cette étude pour améliorer notre connaissance de ces produits, au niveau de l'interface polymère-semi-conducteur et aussi pour mieux connaître les variations de leurs caractéristiques en fonction de la température et des variations du taux d'humidité.

Toutefois, les résultats déjà obtenus permettent de retenir les deux polymères étudiés comme agents passivants ou comme isolants multicouches, puisque leurs propriétés électriques et thermiques sont égales ou supérieures à celles de la silice.

Il convient d'ailleurs de signaler que des essais en vraie grandeur ont été faits :

- d'une part au Laboratoire de Génie Electrique de Toulouse où les polyamides-imides étudiés ont été utilisés pour la protection de circuits intégrés. Les résultats obtenus ont été tout à fait satisfaisants ;

- d'autre part on peut noter que la division semiconducteur d'Hitachi utilise le P.I.Q. comme isolant multicouche et que la Société I.B.M. produit une mémoire DRAM MOS de $64 \mathrm{kbits}$ dont l'isolation est assurée par un polymère thermostable.

Enfin, plusieurs laboratoires japonais ou américains utilisent les polymères thermostables pour l'isolation multicouche ou la passivation. 


\section{Bibliographie}

[1] Beauzee, C., Polymères organiques utilisables à tem pératures élevées (Edition Technip) 1983.

[2] Dubois, J. C., Polymères organiques utilisables à températures élevées (Edition Technip) 1983.

[3] REICH, B., Solid State Technol. (1978) 82.

[4] SnABle, G. L., J. Electrochem. Soc. (1975) 1092.
[5] Wilson, A. M., Thin Solid. Films (1981) 145.

[6] Benalla, H., Thèse Docteur-Ingénieur, Université de Toulouse, 1984.

[7] BuI, A., FARENC, J., Rapport de contrat, Laboratoire de Génie Electrique, 1985. 